Supporting Information for

\title{
Single-Molecule Detection of a Fluorescent Nucleobase Analog via Multiphoton Excitation
}

David Nobis, ${ }^{1}$ Rachel S. Fisher, ${ }^{2}$ Mats Simmermacher, ${ }^{2}$ Patrycja A. Hopkins, ${ }^{3}$ Yitzhak Tor, ${ }^{3}$ Anita C. Jones, ${ }^{2, *}$ and Steven W. Magennis. ${ }^{1, *}$

${ }^{1}$ WestCHEM School of Chemistry, University of Glasgow, Joseph Black Building, University Avenue, Glasgow, G12 8QQ, UK

${ }^{2}$ EaStCHEM School of Chemistry, The University of Edinburgh, Joseph Black Building, David Brewster Road, Edinburgh EH9 3FJ, U.K

${ }^{3}$ Department of Chemistry and Biochemistry, University of California, San Diego, 9500 Gilman Drive, La Jolla, USA

*Correspondence to Prof. Anita Jones (a.c.jones@ed.ac.uk) or Dr. Steven Magennis (steven.magennis@glasgow.ac.uk)

\section{This PDF includes:}

Supplementary Methods

Supplementary Figures S1-S10

Supplementary Tables S1-S4

References 


\section{SUPPLEMENTARY METHODS}

\section{Two-photon spectroscopy and cross section calculations}

The experimental system has been described previously. ${ }^{1}$ A mode-locked Ti:sapphire laser (see description of fluorescence lifetime measurements) was used as the excitation source. Measurements were made at between twenty and thirty different incident powers. Three spectra were collected at each power and an average taken. The incident power was measured using a Coherent FieldMaster power meter. Two photon cross sections were determined by measuring the two-photon brightness of the sample, relative to that of a reference standard, as described previously. ${ }^{1}$ Two two-photon cross section standards were utilised: rhodamine $6 \mathrm{G}$ in methanol and coumarin 153 in DMSO. These standard have been studied previously across a wide range of excitation wavelengths. ${ }^{2}$ The cross-section of rhodamine $6 \mathrm{G}$, at the excitation wavelength, was confirmed by measuring it relative to coumarin 153 in DMSO.

$2 \mathrm{P}$ cross-sections $\left(\sigma_{2}\right)$ were calculated by comparison to a reference:

$$
\frac{\sigma_{2}^{S} \phi^{S}}{\sigma_{2}^{R} \phi^{R}}=\frac{\eta^{R} n^{S} C^{R} F^{S}\left\langle P^{R}\right\rangle^{2}}{\eta^{S} n^{R} C^{S} F^{R}\left\langle P^{S}\right\rangle^{2}}
$$

where $\phi$ is the quantum yield of fluorescence, $\eta$ is a term that accounts for the wavelengthdependent collection efficiency of the fluorescence, $\mathrm{n}$ is the refractive index of the solvent, $\mathrm{C}$ is the concentration, $\mathrm{F}$ is the integrated fluorescence signal from the recorded spectrum, $\mathrm{P}$ is the excitation power, and the superscripts $\mathrm{S}$ and $\mathrm{R}$ refer to either the sample or reference.

Equation 1 can be rewritten as Equation 2: 


$$
\sigma_{2}^{S}=\frac{\sigma_{2}^{R} \phi^{R} n^{S} C^{R}}{\phi^{S} n^{R} C^{S}} \frac{F^{S}\left\langle P^{R}\right\rangle^{2}}{\alpha^{R} F^{R}\left\langle P^{S}\right\rangle^{2}}
$$

where $\alpha^{R}$ is correction factor, equivalent to the ratio of $\frac{\eta^{R}}{\eta^{S}}$ which corrects for the wavelength dependence of the detector and the second term can be calculated from the slope of a plot of integrated emission intensity (F) multiplied by an appropriate correction factor $\left(\alpha^{R}\right)$ against power squared $\left(\left\langle P^{R}\right\rangle^{2}\right)$.

\section{Fluorescence lifetime measurements}

Fluorescence lifetimes were determined using time-correlated single photon counting on an Edinburgh Instruments spectrometer equipped with TCC900 photon counting electronics. A mode-locked Ti:sapphire laser (Coherent Mira pumped by Coherent Verdi), producing pulses of duration $\sim 150 \mathrm{fs}$ at a repetition rate of $76 \mathrm{MHz}$, was used as the excitation source. The pulse repetition rate was reduced to $4.75 \mathrm{MHz}$ using a pulse picker (Coherent 9200) and the light was frequency doubled using a Coherent 5-050 harmonic generator. Fluorescence decay curves were recorded over $50 \mathrm{~ns}, 4096$ channels and collected to a total of 10000 counts in the peak channel. Decays were fitted by iterative re-convolution, assuming a multi-exponential function, given in Equation 3.

$I(t)=\sum_{i=1}^{n} A_{i} \exp \left(\frac{-t}{\tau_{i}}\right)$

where $I$ is the fluorescence intensity as a function of time, $t$, (normalised to the intensity at $t=0$ ); $\tau_{\mathrm{i}}$ is the fluorescence lifetime of the $i$ th decay component and $A_{i}$ is the fractional amplitude (Afactor) of that component. Decays were collected at three emission wavelengths and were analysed globally, with $\tau_{\mathrm{i}}$ as the common parameters, using Edinburgh Instruments software FAST.

The average lifetime, $\langle\tau\rangle$, of the emitting population is related to the average quantum yield, $<\phi>$ determined from steady-state intensity measurements, as given by Equation 4 . 
$<\tau>=\frac{\sum_{i=1}^{n} A_{i} \tau_{i}}{\sum_{i=1}^{n} A_{i}}=\frac{<\phi>}{<k_{r}>}$

where $<k_{\mathrm{r}}>$ is average radiative lifetime of the emitting population.

The fraction of the steady-state emission intensity due to each species $i, S S_{i}$, is given by Equation 5.

$S S_{i}=\frac{A_{i} \tau_{i}}{\sum_{i=1}^{n} A_{i} \tau_{i}}$

\section{Calculation of dark-state population}

The presence of non-emitting species (dark states), i.e. species with fluorescence lifetimes too short to be detected at the experimental time resolution, can be inferred from a discrepancy between the relative steady-state quantum yield and the corresponding relative lifetime. The fractional population of dark states. $A_{0}$, is given by Equation 6 .

$A_{0}=1-\frac{<\phi>_{\text {rel }}}{\left\langle\tau>_{\text {rel }}<k_{r}\right\rangle_{\text {rel }}}$

where $\langle\phi\rangle_{\text {rel }}$ is the relative, average quantum yield (measured, for example in two different solvents), $\langle\tau\rangle_{\text {rel }}$ is the corresponding relative, average lifetime and $\left\langle k_{r}\right\rangle_{\text {rel }}$ the corresponding relative, average radiative rate constant.

For DMA ${ }^{\text {th }} \mathbf{a U}$ in Tris buffer, the fractional dark state population (at an excitation wavelength of $380 \mathrm{~nm}$ ) was calculated from the ratio of the quantum yields in buffer and dioxane, 0.003 and 0.12 , respectively, and the ratio of the corresponding average lifetimes, $1.2 \mathrm{~ns}$ and $1.8 \mathrm{~ns}$, respectively, assuming that $\left\langle k_{r}>\right.$ depends on the square of the refractive index of the solvent (refractive indices of 1.350 and 1.420 , for Tris and dioxane, respectively). This gave a fractional dark state population of $96 \%$. 


\section{Multiphoton microscopy}

For 2P microscopy, the $\mathbf{D M} \mathbf{A}^{\text {th }} \mathbf{a U}$ molecule was dissolved in buffer containing $20 \mathrm{mM}$ Tris (Sigma-Aldrich), $150 \mathrm{mM} \mathrm{NaCl}$ (Fluka) at $\mathrm{pH} 7.8$ after sonication for 20 minutes. For calculation of the concentration by UV-Vis absorption, the extinction coefficient for $\mathbf{D M} \mathbf{A}^{\text {th }} \mathbf{a U}$ in pure water was used. ${ }^{3}$ Prior to adding the sample, the buffer was filtered through activated charcoal to remove fluorescent impurities. Ultrapure water was used for buffer preparation (Direct Q3, Merck Millipore). All solvents and buffers were checked for background fluorescence prior to use.

It was not possible to carry out experiments under single-molecule conditions using dioxane as solvent due to the high background signal from fluorescent impurities.

The home-built setup was described in detail recently. ${ }^{1}$ In brief, the excitation source was a broadband Ti:Sapphire laser with a repetition rate of $80 \mathrm{MHz}$ (Vitara UBB, Coherent). The laser spectrum was centred on $800 \mathrm{~nm}$ with a FWHM of $135 \mathrm{~nm}$; the compressed pulses from the oscillator had a duration of 15 fs. The pulse-shaped beam was focussed onto the sample by a 60x water-immersion objective (UPlanSApo, Olympus). The solution samples were placed on a cover slip (Menzel Gläser, Thermo Scientific) and the solution temperature was controlled with an incubator (Live Cell Instrument, CU-501). All measurements were recorded at $22 \pm 1{ }^{\circ} \mathrm{C}$. Sample fluorescence was collected by the same objective and transmitted through a dichroic mirror (Chroma 675dcspxr) and a short pass filter (Semrock FF01-650/SP), split by a polarising beamsplitter cube (PBS) and detected by two avalanche photodiodes (APD) (MPD PDM 50c and MPD \$PD-050-CTB). The signal was subsequently detected by either a photon-counting card (SPC-132, Becker and Hickl GmbH) for multichannel scalar (MCS) analysis or a hardware correlator (ALV-7002, ALV GmbH) for FCS. For the recording of the MCS a bandpass filter (Semrock FF02-470/100-25) was inserted before the polarizing beamsplitter to further reduce 
background. Additionally, all photons that arrived less than a nanosecond after the excitation pulse were discarded to minimize the contribution from scattered light.

\section{Computational Methods}

The transition energies $\Delta E$ and three-photon absorption cross sections $\left\langle\delta^{3 \mathrm{PA}}\right\rangle$ in Table 1 are calculated via the cubic response function within Time-Dependent Density Functional Theory $(\mathrm{TDDFT})^{4}$ using CAM-B3LYP ${ }^{5} / \mathrm{cc}-\mathrm{pVDZ}{ }^{6,7}$ implemented in molecular electronic structure program Dalton ${ }^{8,9}$. The nuclear geometries are optimised with B3LYP ${ }^{10} / \mathrm{cc}-\mathrm{pVTZ}$ in the same program. The absence of imaginary frequencies in vibrational calculations at the same level of theory confirms that the structures are converged to their equilibrium.

The three-photon absorption cross-sections $\sigma^{3 \mathrm{PA}}$ in Table 1 are calculated from the three-photon absorption probabilities $\left\langle\delta^{3 \mathrm{PA}}\right\rangle$ and transition energies $\Delta E$ by, ${ }^{11,12}$

$$
\sigma^{3 \mathrm{PA}}=N \frac{\pi^{4} \alpha a_{0}^{8}}{c^{2}} \frac{\Delta E^{3}}{27} \frac{2}{\pi \Gamma}\left\langle\delta^{3 \mathrm{PA}}\right\rangle
$$

where $\alpha$ is the fine-structure constant, $a_{0}$ the Bohr radius, and $c$ the speed of light in vacuum. $N$ is a factor that refers to the experimental setup, and $\Gamma$ is the full width at half maximum (FWHM) of a Lorentzian function that describes the lifetime broadening of the spectral line. $\left\langle\delta^{3 \mathrm{PA}}\right\rangle, \Delta E$, and $\Gamma$ are given in atomic units. Here, a single beam experiment and linearly polarised light with $N=4 / 3$ and $\Gamma=0.1 \mathrm{eV}$ were assumed.

A calculation of the first root of $\mathbf{D M A} \mathbf{A}^{\text {th }} \mathbf{a U}$ _B as in Table 1, but with the cc-pVTZ basis set, leads to $\Delta E=3.22 \mathrm{eV},\left\langle\delta^{3 \mathrm{PA}}\right\rangle=1,020 \times 10^{6}$ a.u., and $\sigma^{3 \mathrm{PA}}=70.3 \times 10^{-82} \mathrm{~cm}^{6} \mathrm{~s}^{2}$ photon ${ }^{-2}$. The calculation took around 110.8 hours on a node with 16 cores. The calculation of the first ten roots with the cc-pVDZ basis set, in contrast, took 125.3 hours on the same type of machine. Usage of a larger basis set than cc-pVDZ for the full calculation is thus prohibited by the large 
number of roots and the limits of the available computational resources. Besides, the changes of $\Delta E,\left\langle\delta^{3 \mathrm{PA}}\right\rangle$, and $\sigma^{3 \mathrm{PA}}$ are probably smaller than the errors from TDDFT itself and seem negligible from a qualitative perspective.

\section{SUPPLEMENTARY FIGURES}

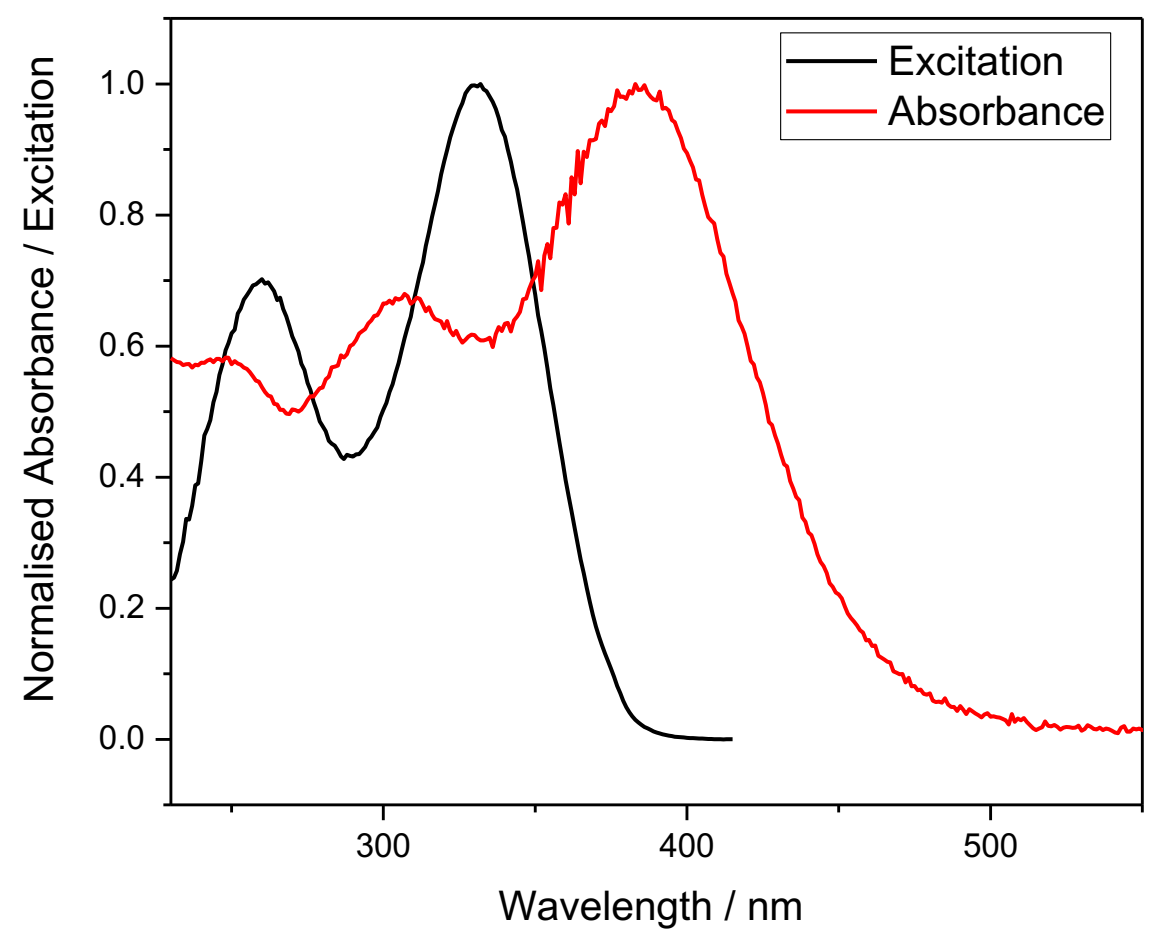

Figure S1. Absorption spectrum (red) and excitation spectrum (black), recorded at an emission wavelength of $430 \mathrm{~nm}$, of $\mathbf{D M} \mathbf{A}^{\text {th }} \mathbf{a} \mathbf{U}$ in Tris buffer. 


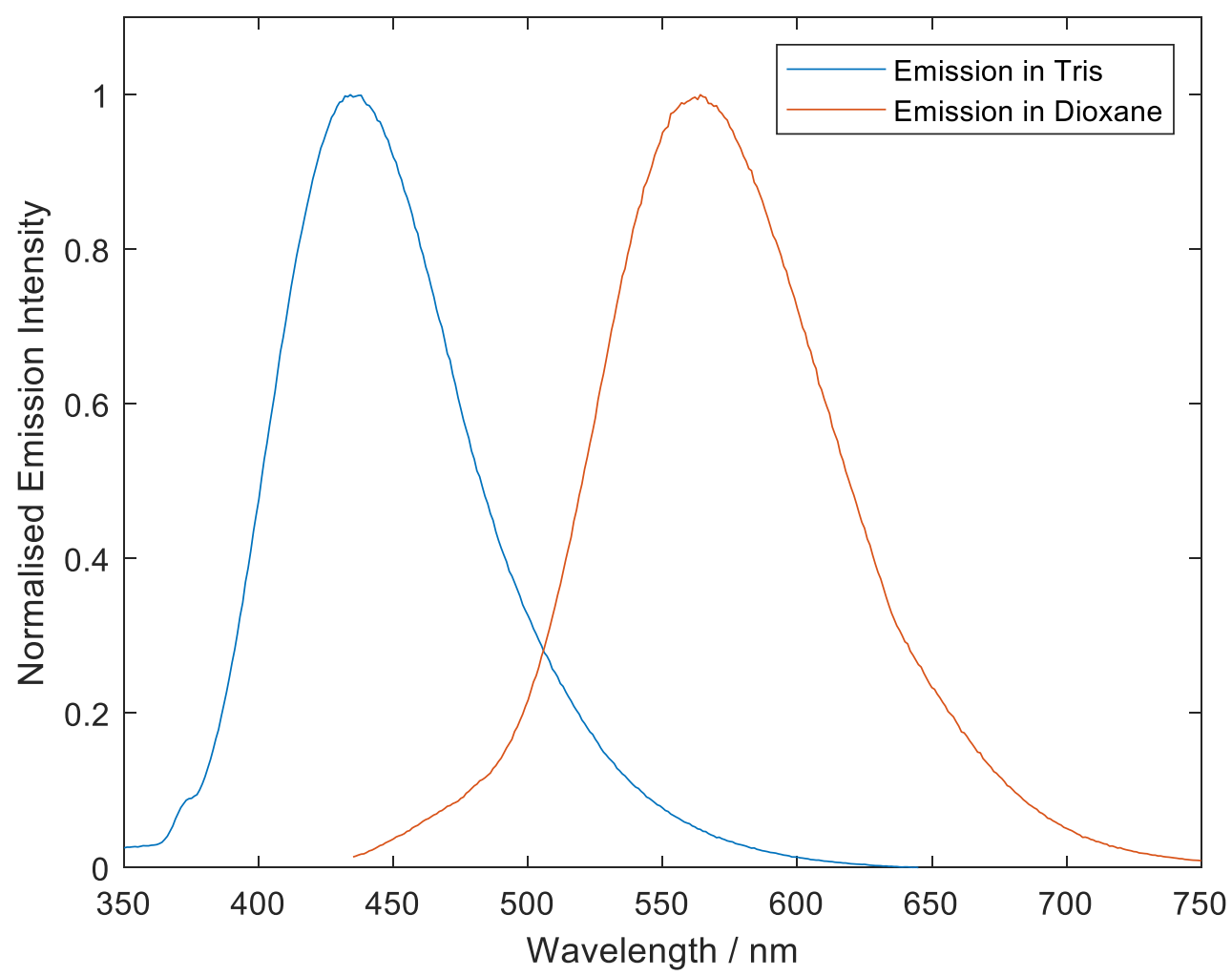

Fig. S2. Emission spectra for $\mathbf{D M A}{ }^{\text {th }} \mathbf{a U}$ in Tris buffer (blue), excitation at $330 \mathrm{~nm}$, and dioxane (orange) excitation at $420 \mathrm{~nm}$. 


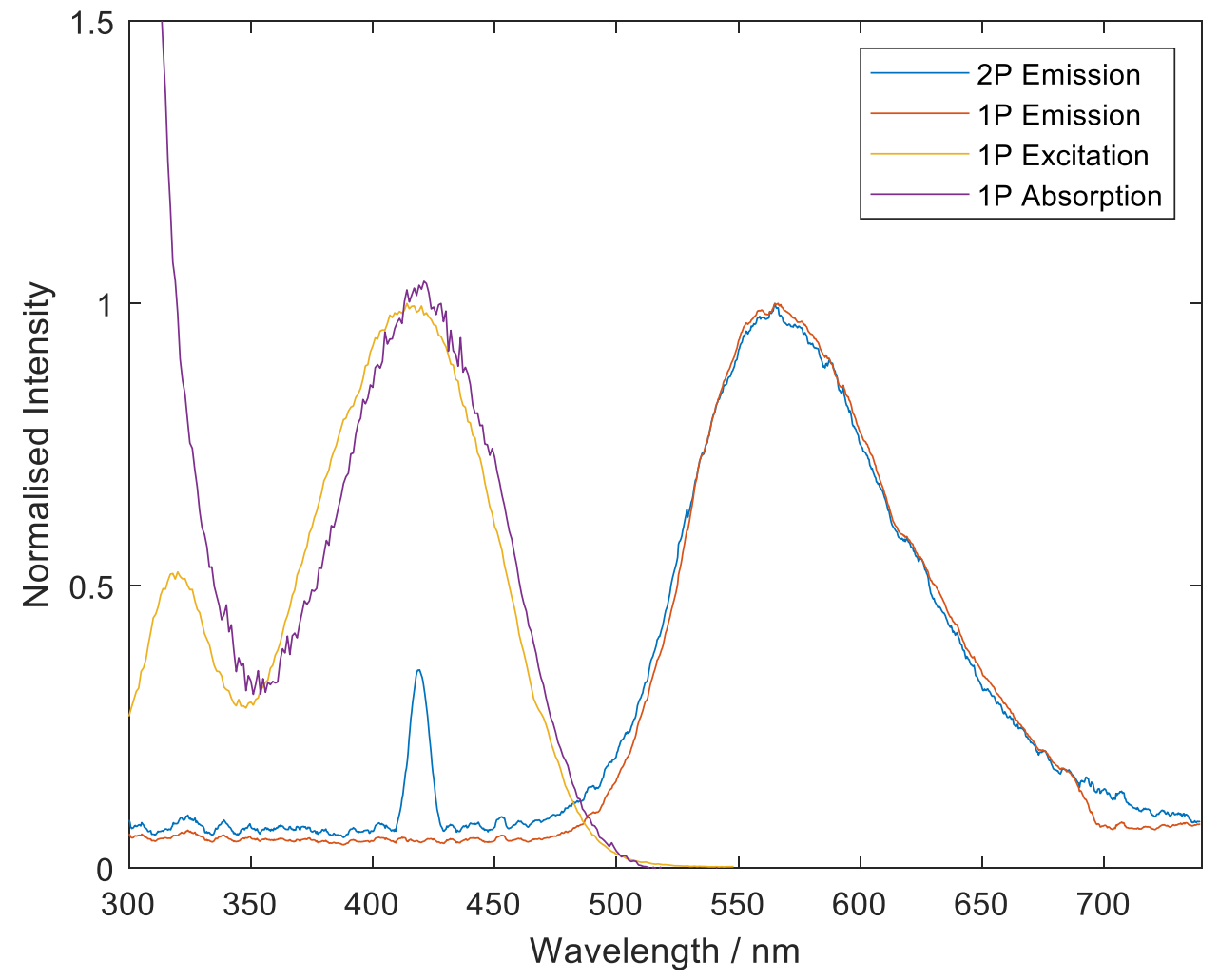

Fig. S3 One-photon excitation (yellow) and absorption (purple) spectra for $\mathbf{D M A} \mathbf{A}^{\text {th }} \mathbf{a U}$ in dioxane, and emission spectra following one-photon (orange) and two-photon (blue) excitation. 


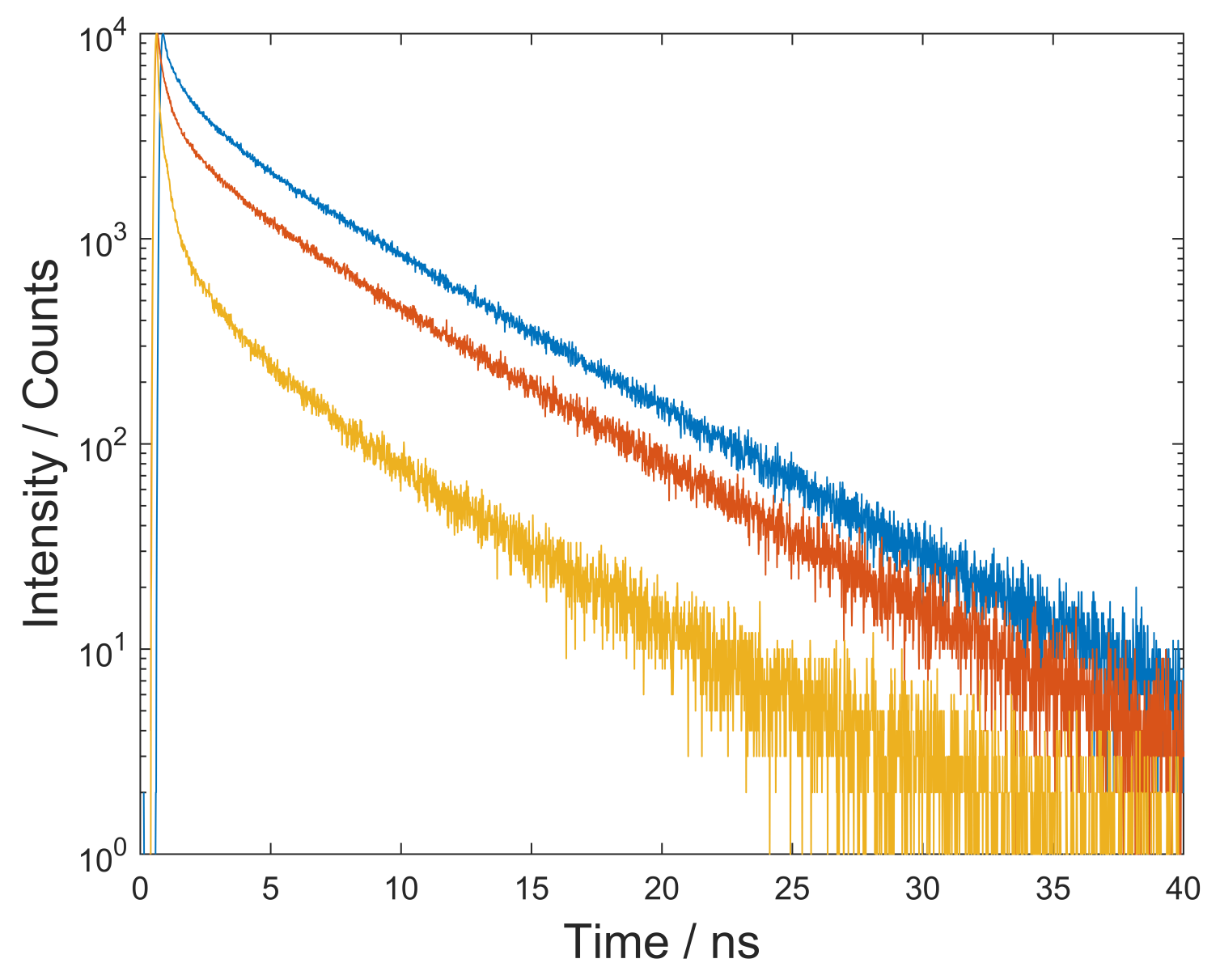

Figure S4. Fluorescence decays of $\mathbf{D M A}{ }^{\text {th }} \mathbf{a U}$ recorded in Tris buffer at excitation wavelengths of $364 \mathrm{~nm}$ (blue), $380 \mathrm{~nm}$ (orange) and $400 \mathrm{~nm}$ (yellow), all at an emission wavelength of 470 nm. 


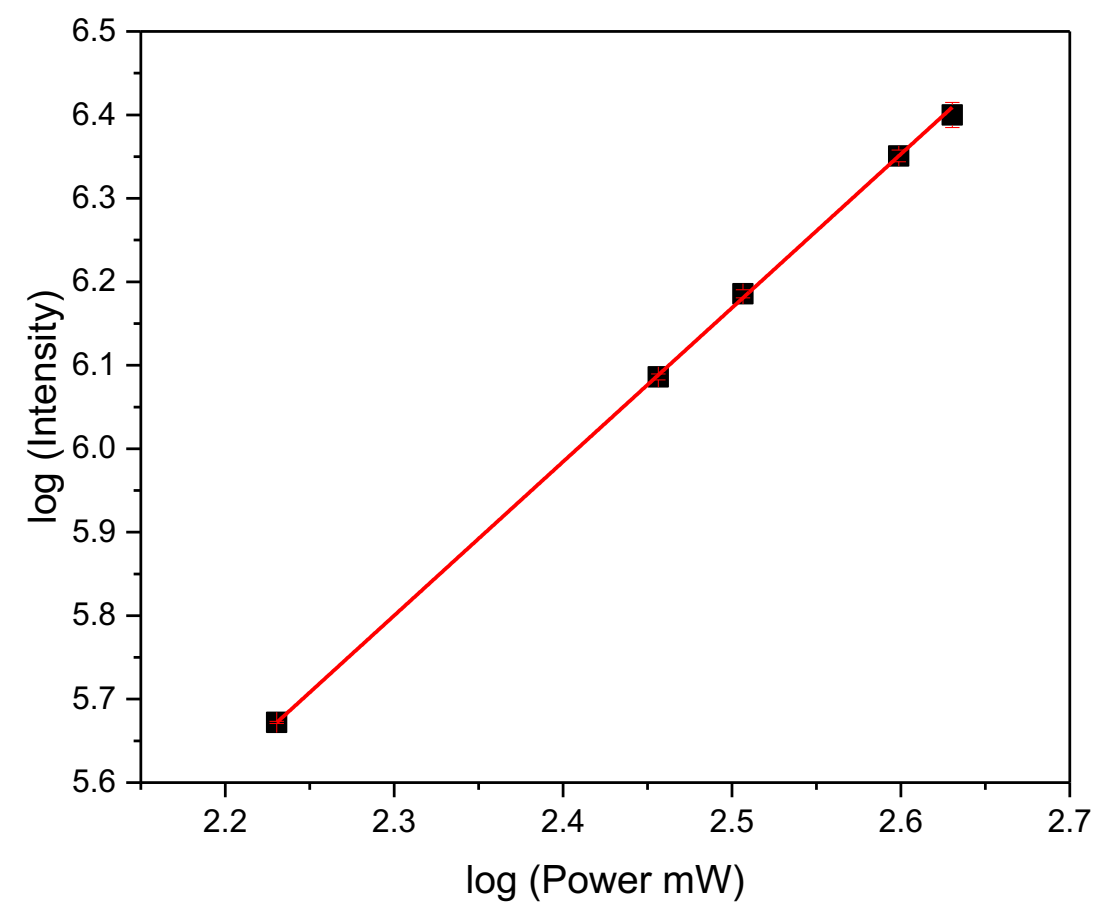

Fig. S5 Logarithmic dependence of the fluorescence intensity vs laser power for $\mathbf{D M} \mathbf{A}^{\text {th }} \mathbf{a} \mathbf{U}$ in dioxane. Excitation wavelength was $840 \mathrm{~nm}$. Laser power was varied from $170 \mathrm{~mW}$ to $427 \mathrm{~mW}$. 


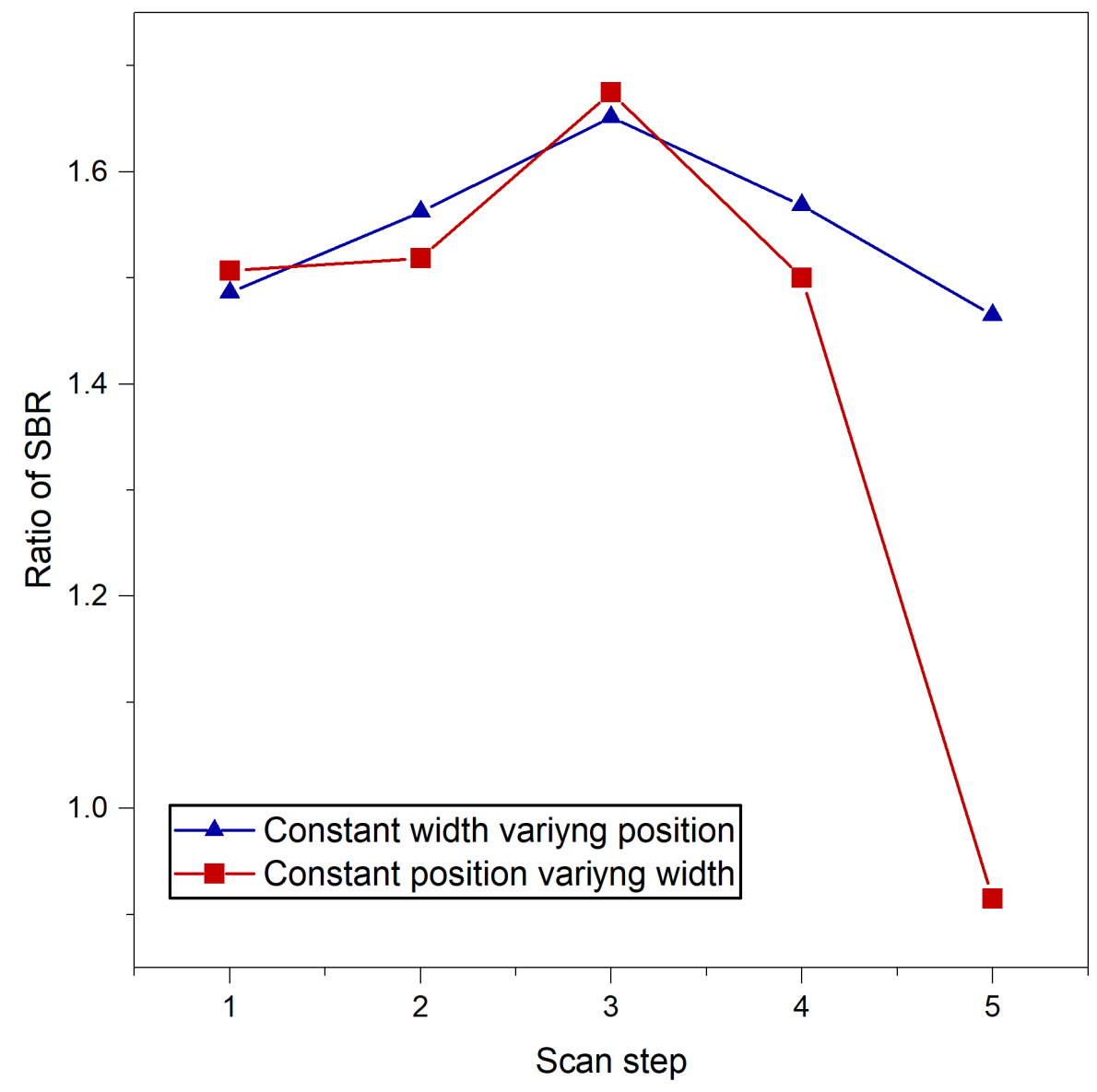

Figure S6. The ratio of the SBR for a compressed pulse at $14 \mathrm{~mW}$ and a pulse with additional spectral amplitude masking. The blue triangles depict the value for a mask with constant width but varying position and the red squares depict the value for constant position but varying width. Scan step 3 results in the same pulseshape for both measurements. 


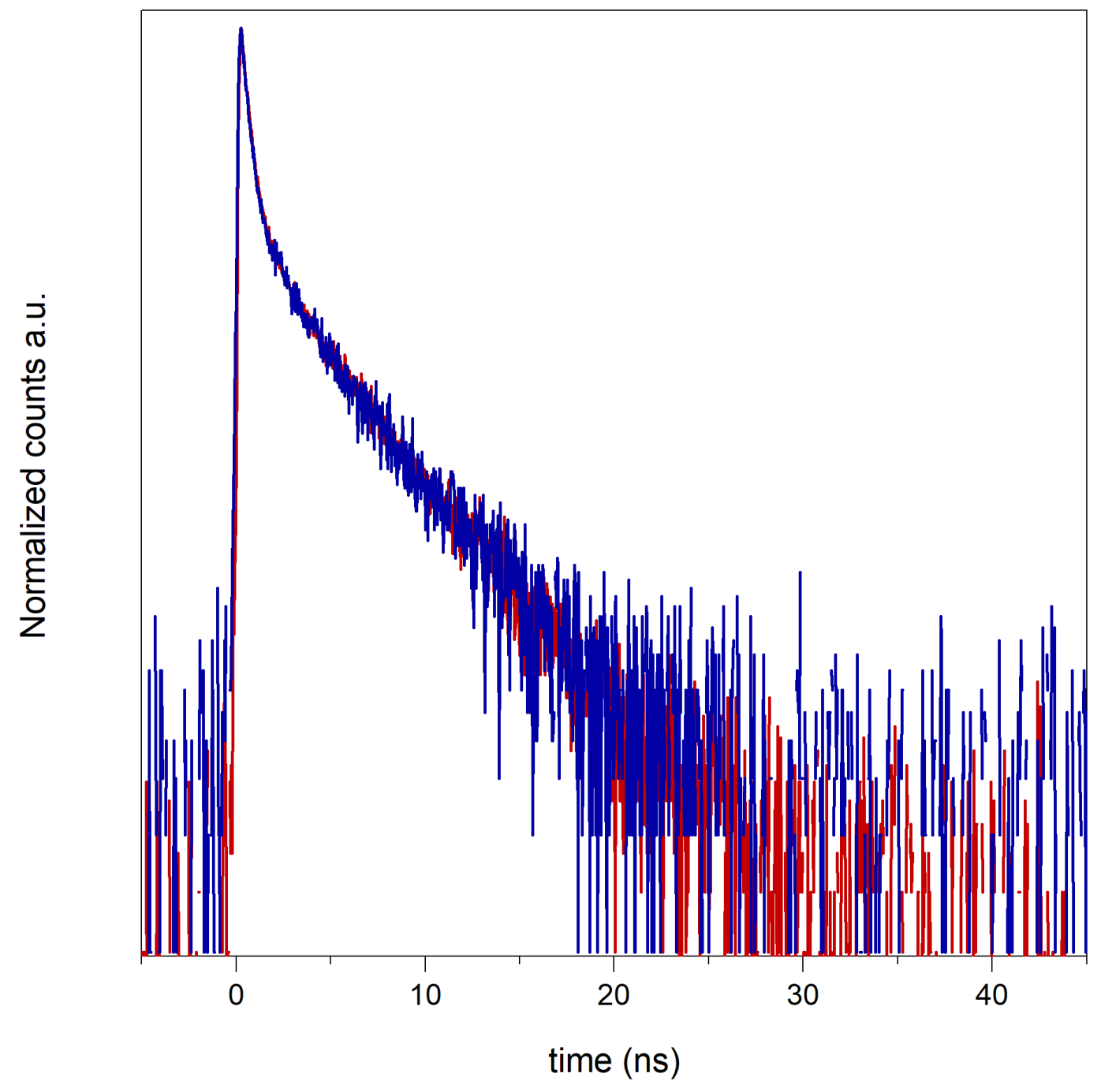

Figure S7. Fluorescence decays of $\mathbf{D M A}{ }^{\text {th }} \mathbf{a U}$ in Tris buffer, measured in the $2 \mathrm{P}$ regime (blue line) and $3 \mathrm{P}$ regime (red line). The laser power was $0.15 \mathrm{~mW}(30 \mathrm{pJ}$ pulse energy) in the $2 \mathrm{P}$ regime and $0.3 \mathrm{~mW}(60 \mathrm{pJ}$ pulse energy) in the $3 \mathrm{P}$ regime. Typical fit parameters, from a tail fit, are $0.40 \mathrm{~ns}(0.76), 1.94 \mathrm{~ns}(0.12)$ and $5.23 \mathrm{~ns}(0.12)$. 

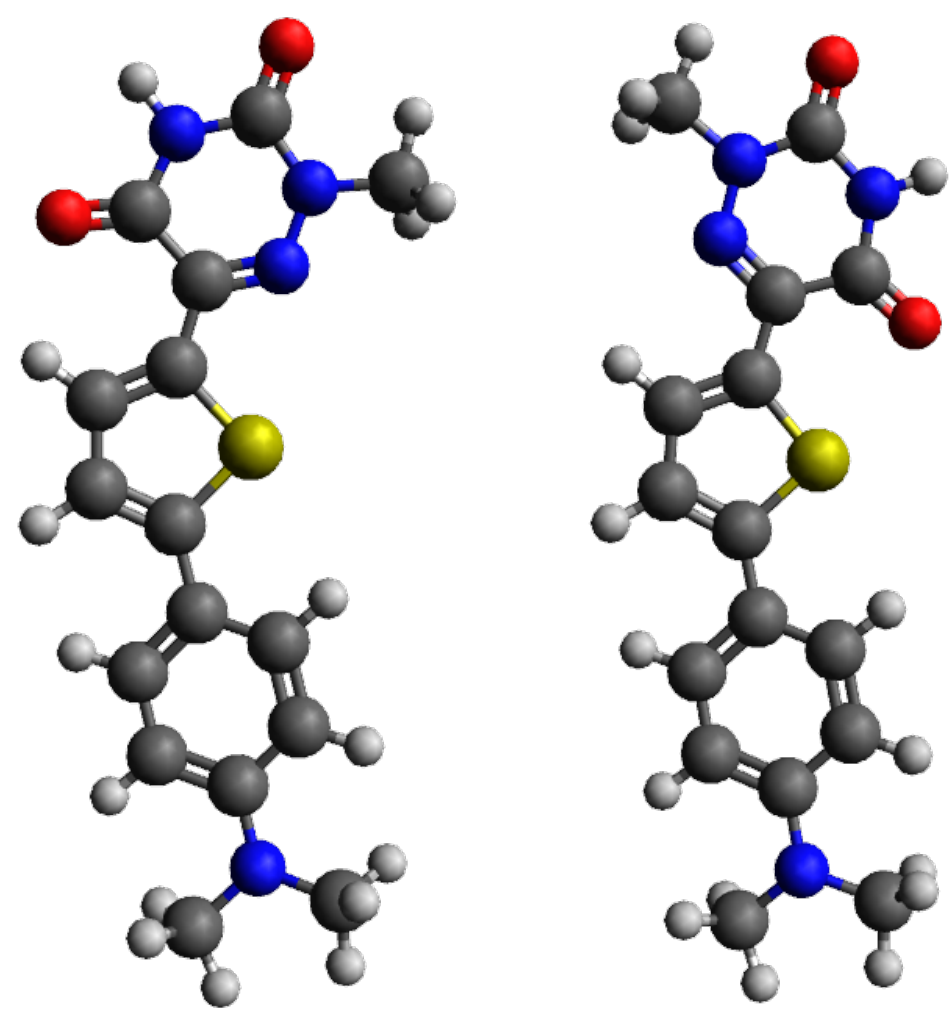

Figure S8. Structures of rotamers $\mathbf{D M} \mathbf{A}^{\text {th }} \mathbf{a U} \_$A (left) and $\mathbf{D M A}{ }^{\text {th }} \mathbf{a} \mathbf{U}_{-}$B (right) optimized with B3LYP/cc-pVTZ. 


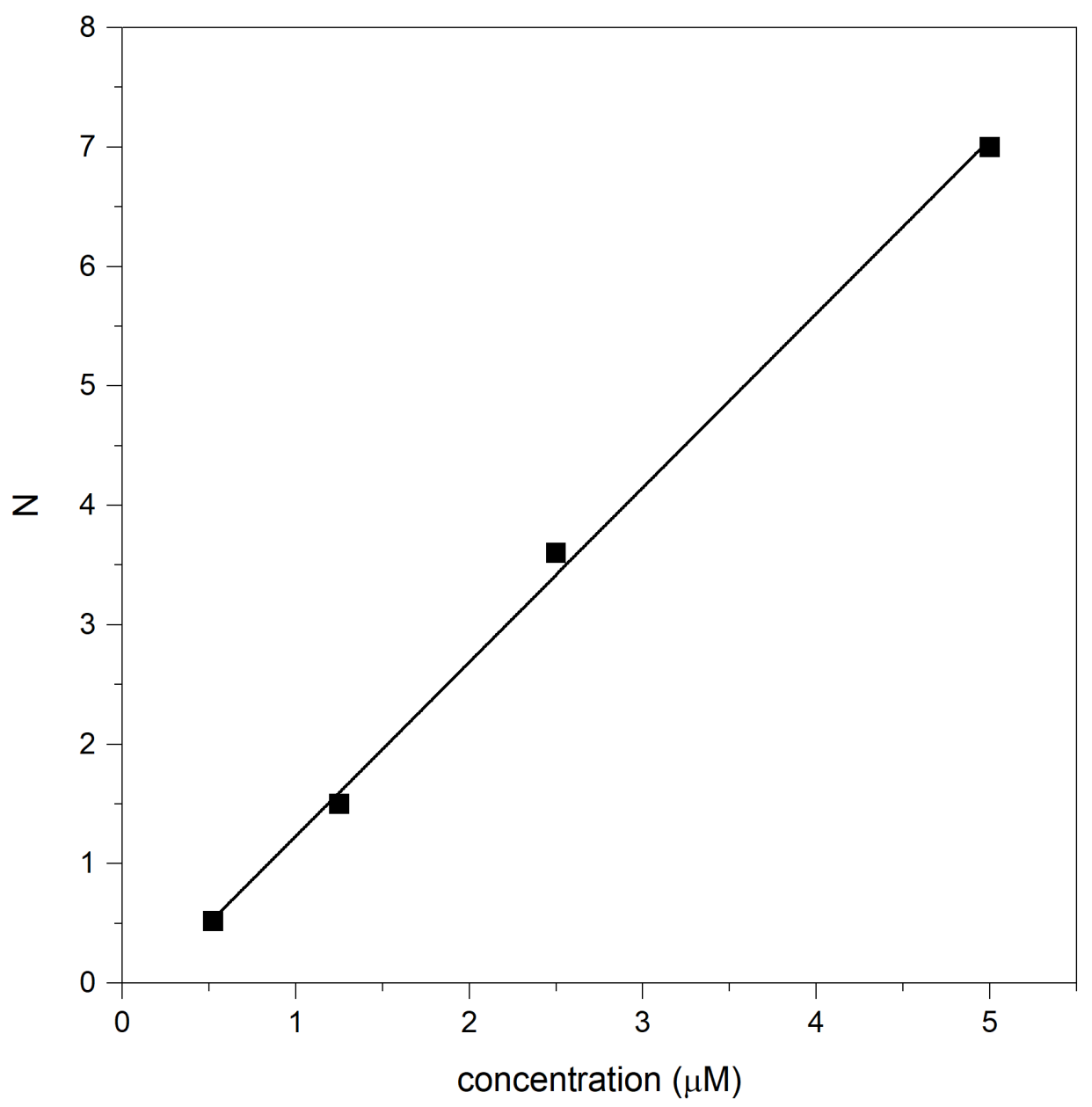

Figure S9. Measured number of $\mathbf{D M} \mathbf{A}^{\text {th }} \mathbf{a U}$ molecules in Tris buffer in the focus of the laser as a function of the sample concentration. The intercept of the fitted line is reasonably close to zero at $-0.2 \pm 0.1$. 


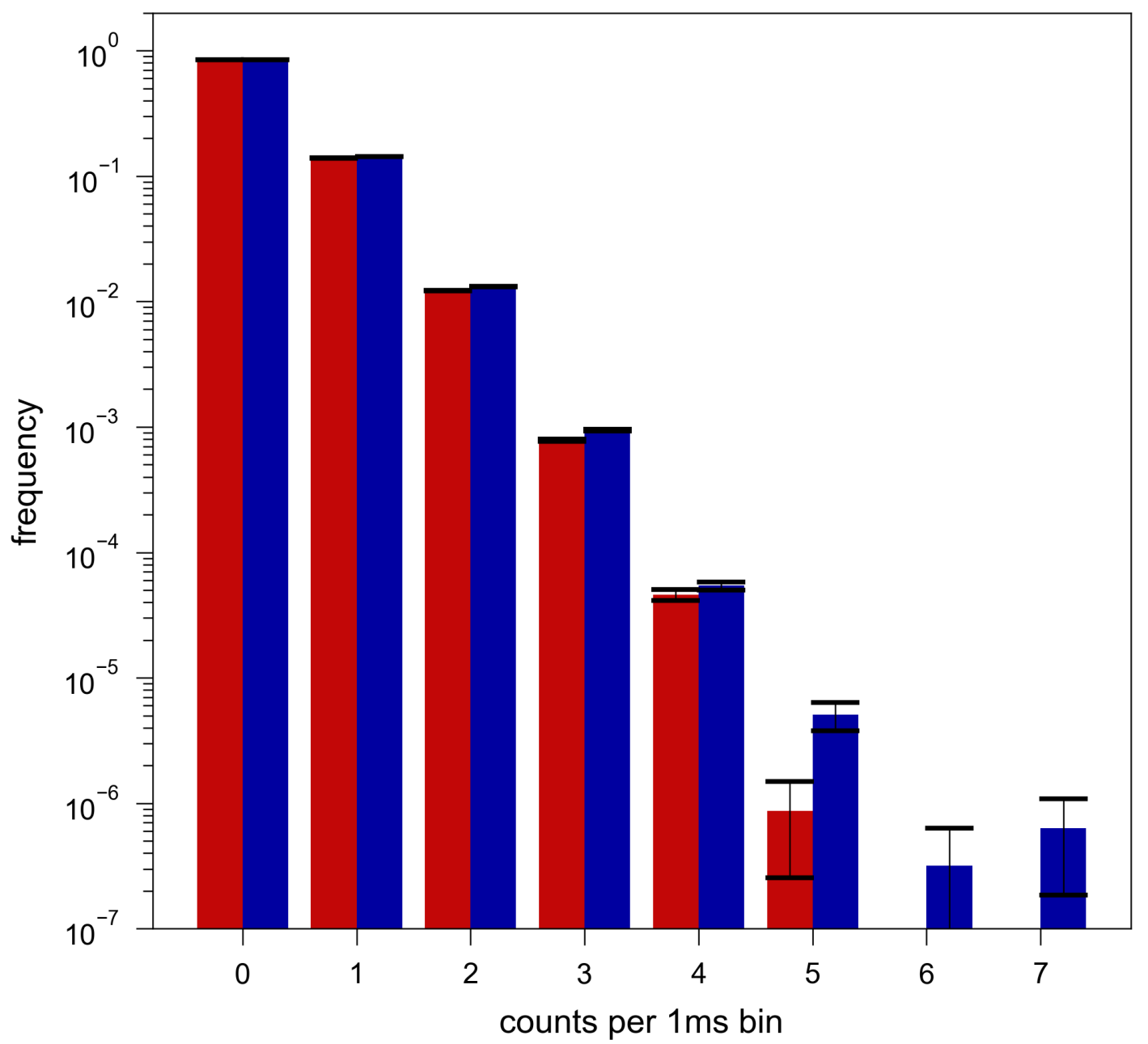

Figure S10. Photon-counting histogram for the single-molecule experiment with $\mathbf{D M A} \mathbf{A}^{\text {th }} \mathbf{a U}$. The red bars show the frequency of events for the background (pure buffer) and the blue bars show the frequency of events for a $\mathbf{D M A}^{\text {th }} \mathbf{a U}$ solution $\left(\sim 10^{-11} \mathrm{M}\right.$ of bright DMAthaU molecules). The frequencies were calculated by dividing the total number of each event by the total number of ms measured over the course of all measurements. They depict the experimental photon-counting probability density. The sample and buffer were recorded under identical measurement conditions. The histograms represent two independent measurements of both buffer and sample, which were then combined, giving a total recording time of $2522.2 \mathrm{~s}$ for buffer and $3387.0 \mathrm{~s}$ for the sample. The error bars depict the statistical uncertainty estimated with the method given in ref. 13 . 


\section{SUPPLEMENTARY TABLES}

Table S1. Quantum yield of $\mathbf{D M A} \mathbf{A}^{\text {th }} \mathbf{a U}$ in Tris buffer measured as a function of excitation wavelength between 380 and $300 \mathrm{~nm}$.

\begin{tabular}{|l|l|}
\hline Wavelength / nm & Quantum Yield \\
\hline 380 & 0.003 \\
\hline 370 & 0.007 \\
\hline 360 & 0.017 \\
\hline 350 & 0.032 \\
\hline 340 & 0.048 \\
\hline 330 & 0.053 \\
\hline 320 & 0.043 \\
\hline 310 & 0.031 \\
\hline 300 & 0.023 \\
\hline
\end{tabular}

Table S2. Decay parameters for DMA ${ }^{\text {thaU }}$ in Tris buffer obtained from global analysis (common lifetimes) of decays recorded at several emission wavelengths, at an excitation wavelength of $360 \mathrm{~nm}$. Parameters given are: fluorescence lifetime $\left(\tau_{\mathrm{i}}\right)$, fractional population, $\left(A_{\mathrm{i}}\right)$ contribution to steady-state intensity $\left(S S_{\mathrm{i}}\right)$ and number-average lifetime $(<\tau>)$.

\begin{tabular}{|c|c|c|c|c|c|c|c|c|c|c|}
\hline $\begin{array}{l}\text { Emission } \\
\text { Wavelength } \\
\text { / nm }\end{array}$ & $\tau_{1} / \mathrm{ns}$ & $\begin{array}{l}\tau_{2} / \\
\mathrm{ns}\end{array}$ & $\begin{array}{l}\tau_{3} / \\
\mathrm{ns}\end{array}$ & $\mathrm{A}_{1}$ & $\mathrm{~A}_{2}$ & $\mathrm{~A}_{3}$ & $\mathrm{SS}_{1}$ & $\mathrm{SS}_{2}$ & $\mathrm{SS}_{3}$ & $\begin{array}{l}<\tau>/ \\
\mathrm{ns}\end{array}$ \\
\hline 400 & \multirow{5}{*}{0.19} & \multirow{5}{*}{1.3} & \multirow{5}{*}{5.9} & 0.35 & 0.36 & 0.29 & 0.03 & 0.21 & 0.76 & 2.27 \\
\hline 430 & & & & 0.37 & 0.30 & 0.33 & 0.03 & 0.17 & 0.80 & 2.43 \\
\hline 450 & & & & 0.38 & 0.29 & 0.33 & 0.03 & 0.16 & 0.81 & 2.40 \\
\hline 470 & & & & 0.44 & 0.26 & 0.30 & 0.04 & 0.16 & 0.80 & 2.19 \\
\hline 490 & & & & 0.52 & 0.24 & 0.24 & 0.05 & 0.17 & 0.78 & 1.86 \\
\hline
\end{tabular}


Table S3. Decay parameters for DMA thaU in Tris buffer obtained from global analysis (common lifetimes) of decays recorded at three excitation wavelengths, 360, 380 and 400 $\mathrm{nm}$, at an emission wavelength of $470 \mathrm{~nm}$. The quantum yield $(\phi)$ from steady-state measurements is also shown. There is a marked dependence of the A-factors on excitation wavelength. The shortest lifetime accounts for $44 \%$ of the emitting population when excited at $360 \mathrm{~nm}$, increasing to $81 \%$ when excited at $400 \mathrm{~nm}$. There is a corresponding decrease in the fractional population of the longest lifetime component from $30 \%$ to $4 \%$. This results in a decrease in the average lifetime from $2.2 \mathrm{~ns}$ to $0.43 \mathrm{~ns}$, on increasing the excitation wavelength from 360 to $400 \mathrm{~nm}$. However, the decrease in average lifetime is not commensurate with the decrease in quantum yield; $\phi$ decreases by a factor of 6 on increasing the excitation wavelength from 360 to $380 \mathrm{~nm}$, whereas $\langle\tau\rangle$ decreases by a factor of less than 2. This is consistent with the excitation of dark states at longer wavelengths.

\begin{tabular}{|l|l|l|l|l|l|l|l|l|}
\hline $\begin{array}{l}\text { Excitation } \\
\text { Wavelength } \\
/ \mathrm{nm}\end{array}$ & $\tau_{1} / \mathrm{ns}$ & $\tau_{2} / \mathrm{ns}$ & $\tau_{3} / \mathrm{ns}$ & $\mathrm{A}_{1}$ & $\mathrm{~A}_{2}$ & $\mathrm{~A}_{3}$ & $<\tau>/ \mathrm{ns}$ & $\phi$ \\
\hline 360 & \multirow{2}{*}{0.19} & 1.3 & 5.9 & 0.44 & 0.26 & 0.30 & 2.2 & 0.017 \\
\cline { 1 - 6 } & & & & 0.66 & 0.19 & 0.15 & 1.2 & 0.003 \\
\cline { 1 - 6 } & & & & 0.81 & 0.15 & 0.04 & 0.60 & - \\
\hline
\end{tabular}

\section{Table S4.}

Decay parameters for $\mathbf{D} \mathbf{M A}^{\text {thaU }} \mathbf{U}$ in dioxane obtained from global analysis (common lifetimes) of decays recorded at three emission wavelengths, at an excitation wavelength of $360 \mathrm{~nm}$.

\begin{tabular}{|l|l|l|l|l|l|}
\hline $\begin{array}{l}\text { Emission } \\
\text { Wavelength } \\
/ \mathrm{nm}\end{array}$ & $\tau_{1} / \mathrm{ns}$ & $\tau_{2} / \mathrm{ns}$ & $\mathrm{A}_{1}$ & $\mathrm{~A}_{2}$ & $<\tau>/ \mathrm{ns}$ \\
\cline { 1 - 4 } 545 & \multirow{3}{*}{0.40} & \multirow{2}{*}{3.6} & 0.37 & 0.63 & 1.8 \\
\hline 565 & & 0.37 & 0.63 & 1.8 \\
\hline 585 & & 0.39 & 0.61 & 1.8 \\
\hline
\end{tabular}

\section{REFERENCES}

(1) Fisher, R. S.; Nobis, D.; Fuchtbauer, A. F.; Bood, M.; Grotli, M.; Wilhelmsson, L. M.; Jones, A. C.; Magennis, S. W. Pulse-Shaped Two-Photon Excitation of a Fluorescent Base Analogue Approaches Single-Molecule Sensitivity. Phys. Chem. Chem. Phys. 2018, 20, 28487-28498.

(2) de Reguardati, S.; Pahapill, J.; Mikhailov, A.; Stepanenko, Y.; Rebane, A. HighAccuracy Reference Standards for Two-Photon Absorption in the 680-1050 nm Wavelength Range. Opt. Express 2016, 24, 9053-9066.

(3) Hopkins, P. A.; Sinkeldam, R. W.; Tor, Y. Visibly Emissive and Responsive Extended 6-Aza-Uridines. Org. Lett. 2014, 16, 5290-5293.

(4) Jansik, B.; Sałek, P.; Jonsson, D.; Vahtras, O.; Ågren, H. Cubic Response Functions in Time-Dependent Density Functional Theory. J. Chem. Phys. 2005, 122, 054107. 
(5) Yanai, T.; Tew, D. P.; Handy, N. C. A New Hybrid Exchange-Correlation Functional using the Coulomb-Attenuating Method (CAM-B3LYP).Chem. Phys. Lett. 2004, 393, 51-57.

(6) Dunning, T. H. Gaussian-Basis Sets For Use In Correlated Molecular Calculations. 1. The Atoms Boron Through Neon Ans Hydrogen. J. Chem. Phys. 1989, 90, 1007-1023.

(7) Woon, D. E.; Dunning, T. H. Gaussian-Basis Sets For Use In Correlated Molecular Calculations. 3. The Atoms Aluminum Through Argon. J. Chem. Phys. 1993, 98, 13581371.

(8) Aidas, K. et al. The Dalton Quantum Chemistry Program System. WIREs-Comput. Mol. Sci. 2014, 4, 269-284.

(9) Dalton, a molecular electronic structure program, Release v2019.alpha （2018), see http://daltonprogram.org.

(10) Becke, A. D. Density-Functional Thermochemistry .3. The Role of Exact Exchange. $J$. Chem. Phys. 1993, 98, 5648-5652.

(11) Cronstrand, P.; Jansik, B.; Jonsson, D.; Luo, Y.; Ågren, H. Density Functional Response Theory Calculations of Three-Photon Absorption. J. Chem. Phys. 2004, 121, 9239-9246.

(12) Friese, D. H.; Beerepoot, M. T. P.; Ringholm, M.; Ruud, K. Open-Ended Recursive Approach for the Calculation of Multiphoton Absorption Matrix Elements. J. Chem. Theory Comput. 2015, 11, 1129-1144.

(13) Chen, Y.; Müller, J. D.; So, P. T. C.; Gratton, E. The Photon Counting Histogram in Fluorescence Fluctuation Spectroscopy. Biophys. J. 1999, 7, 553-567. 